\title{
The research of new start frame beginning spinning position signal acquisition system based on reed sensor
}

\author{
Qingbin Zhao ${ }^{1, a}$, Guangdong Jiang ${ }^{1, b}$ \\ ${ }^{1}$ School of Medical Physics, Weifang Medical University, Shandong 261053, China \\ ajianggd@wfmc.edu.cn, byzhb@wfmc.edu.cn
}

Keywords: Spinning, Signal acquisition, reed sensors.

\begin{abstract}
In this paper, a new frame beginning spinning position signal acquisition system is designed with reed sensors. Compared with the beginning spinning position signal acquisition device, the experiment proves that, the structure of the signal acquisition device is easy to adjust, stable and long usage life, and processing, manufacturing cost is low and facilitate to Promotion.
\end{abstract}

\section{Introduction}

At present, the frame beginning spinning position signal acquisition system is made up through the worm shaft semicircle metal CAM installed on the worm shaft below L hold claw institutions and its corresponding travel switch installed on the base of the frame head, the travel switch is designed as signal device connected to a frame of control system[1-5]. In the process of spinning, semicircle metal CAM always contact with the travel switch which has been closed and sending a high level signals, it means the frame running normally. At the end of the yarn, travel switch contact with semicircle metal CAM disconnected, the spinning complete the control spinning process. As the existing switch is contact form, and always contact with semicircle metal CAM phase in the entire spinning process, the switch is easy to damage. To solve the problems, a new frame beginning spinning position signal acquisition system is designed with reed sensors, which is Long service life, convenient maintenance and low cost.

\section{Signal acquisition device design scheme}

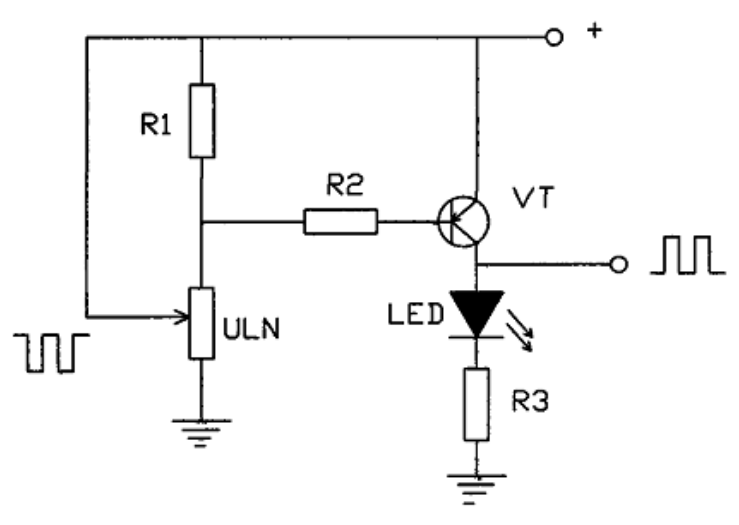

Figure1 Hall sensor circuit diagram

In order to improve the stability of the textile spinning, we had tried to put forward the Hall sensor based start spinning position signal acquisition system. The hall sensor is a cylindrical device, which has a threaded surface and two nuts for fixing the Hall sensor, which ends with a cable and LED indicator. The Hall sensor circuit is showed in Figure 1, the Hall sensor comprises a pulse switch VT, a first resistor R1, a second resistor R2, a third resistor R3, a light emitting diode LED, the Hall element ULN, the emitter of pulse switch VT connected to the supply, the collector is output terminal, the base via a second resistor R2 and the Hall element ULN connected to the ground, a 
second resistor R2 and the Hall element ULN contact through the first resistor R1 is connected to power, pulse switch set VT electrode by a light emitting diode LED, a third resistor R3 to ground.

When the permanent magnet closed to Hall sensor, the low level signal is always inverted high signal output by the control system to achieve the spinning frame doffing position to achieve the parking requirements, while at the end of a series of light-emitting diode (LED) lights. This improvement can be slightly modified spinning machine stability, but in our follow-up study of make adjustments to sensors this can more efficient to improve the stability.

Frame start spinning position signal acquisition device is shown in figure2, among them 1 is fixed screw, 2 is worm shaft, 3 is collar, 4 is nut (figure 3), 5 is adjustment screw, 6 is semicircle CAM, 7 is permanent magnets, 8 is reed sensor, 9 is support, 10 is cable (figure 4)

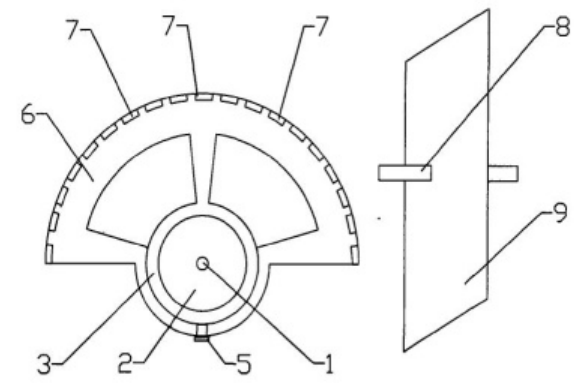

Figure 2 signal acquisition device structure diagram

This paper adopts the following technical scheme: the beginning of the frame spinning position signal acquisition device, including semicircle CAM (6), the signal acquisition sensor (8), semicircular CAM installed at the bottom of the car frame level rise hold claw mechanism worm shaft (2), the location of the sensor signal acquisition corresponding to the semicircle CAM, sensor signal acquisition electrical connected to control system , and semicircle CAM is made of nylon materials, the semicircular arc of the CAM is equipped with a number of uniform distribution of permanent magnet (7), and the sensor is reed sensor signal collection. At the beginning of the spinning frame spinning, the permanent magnet on the semicircle nylon CAM circular can induct the control signal send by reed sensor. As the distance between two permanent magnets is small, reed sensor moving between two permanent magnets can guarantee a stable signal output, which can control system frame normal driving.

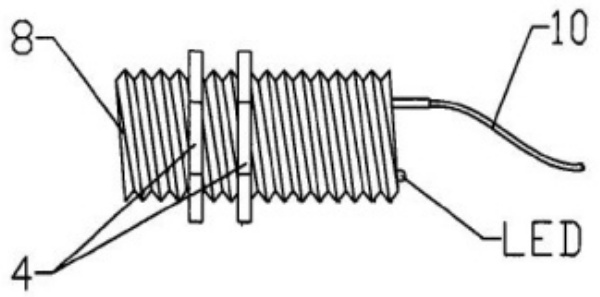

Figure 3 reed sensor structure diagram 


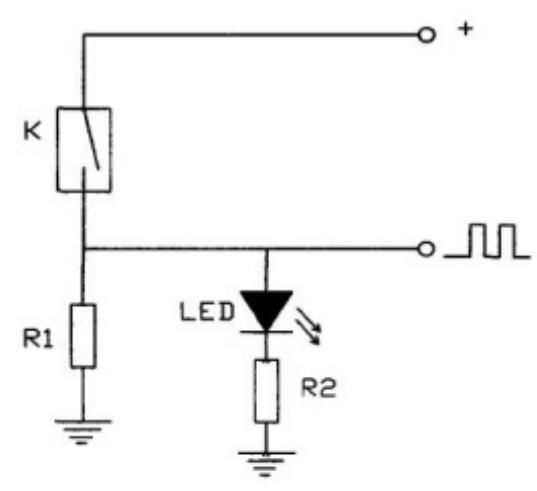

Figure 4 reed sensor circuit diagram

The reed sensor is also optimized. As shown in figure 3, the reed sensor is a cylindrical device, its surface with thread used to fix two nut(4) of the reed pipe sensor, its department with cables and LED lights. Reed pipe specific circuit is shown in figure 4, reed sensor including a pulse switch $\mathrm{K}$, the first resistor R1, the second resistor R2, LED light-emitting diodes. The pulse switch tube $\mathrm{K}$ and the first resistor R1 is connected between power supply and ground, its connection point is output, and the other end of the pulse switch tube $\mathrm{K}$ is connected to the power, the other end of the first resistance $\mathrm{Rl}$ is connected to the ground, the LED and R2 is in series, the other side of LED is output side, the other end of $\mathrm{R} 2$ is connected to the ground.

When permanent magnet in close to reed sensor, the pulse switch tube $\mathrm{K}$ is in conduction, its output in high level, which can be used to realization frame start spinning position parking demands by controlling system, and the LED lamp lit at the same time.

\section{System operation and analysis}

As shown in figure2, the frame spinning position signal acquisition device, including the $86 \mathrm{~mm}$ radius semicircle CAM made of nylon materials. The semicircular CAM installed at the bottom of the car frame level rise hold claw mechanism worm shaft, between semicircle CAM and axle is screw used to adjust semicircle CAM Angle to reach the purpose of parking spinning frame at spinning start position signal. The shaft sleeve is in the worm wheel at the bottom of the Level rise hold claw by the fixed screws. There are 19 permanent magnets embedded on the arc of the semicircular CAM evenly distributed, the diameter of the permanent magnet is $8 \mathrm{~mm}$, and the distance between two permanent magnets is small. When reed sensor moves between two permanent magnets, the small distance can guarantee stable signal output. The position of reed sensor installed on the stents is corresponding to permanent magnets of semicircle CAM , and reed sensor is connected to the electric control system. When permanent magnet in close to reed sensor, the pulse switch tube $\mathrm{K}$ is in conduction, its output in high level, which can be used to realization frame start spinning position parking demands by controlling system, and the LED lamp lit at the same time.

Due to the adjustment of the reed sensor distance is big, therefore in the process of using it is easy to adjust, at the same time, dry reed pipe sensor good anti-jamming, stable performance, long service life, low price, easy to promote. The nylon semicircular CAM only have the effect of supporting permanent magnet, it is easy processing and low manufacturing cost. Compared with travel switch type of spinning position signal acquisition, this reed pipe sensor can reduce 100 yuan costs.

\section{Conclusion}

In this paper, a new frame beginning spinning position signal acquisition system is designed with reed sensors. Compared with the beginning spinning position signal acquisition device, the 
experiment proves that, the structure of the signal acquisition device is easy to adjust, stable and long usage life, and processing, manufacturing cost is low and facilitate to Promotion.

\section{Reference}

[1] W.L.DIAO: The performance of a DTMl 29 type frame and spinning practice(Cotton Textile Technology, China2009) ,P.43-45.(In Chinese)

[2] D.S.LIAO: The application of variable frequency speed regulation technology in spinning frame (Cotton Textile Technology, China2003. ,p.261-264.(In Chinese)

[3] Z.L. ZHAI,T.X. GAO and D.S.YAO: The application of textile intelligence frequency converter in spinning frame(Cotton TextileTechnology, China 2007) ,p.106-107.(In Chinese)

[4] L.G.ZHANG: The practice of the frame in spinning fine yarn of before area equipped with pressure bar (CottonTextile Technology, China2008,) ,p.38-39.(In Chinese)

[5] J.F.Shao, L.S.Qin: DeVelopment of computer monitoring system of spinning frame based on C/S mode(Journal of Textile Research,China2009),p126-130. (In Chinese) 